\title{
Interleukin-12 cDNA skin transfection potentiates human papillomavirus E6 DNA vaccine-induced antitumor immune response
}

\author{
Jun Tan, ${ }^{1,2}$ Ning-Sun Yang, ${ }^{3}$ Joel G. Turner, ${ }^{1,2}$ Gui-Lian Niu, ${ }^{1,2}$ Hunein F. Maassab, ${ }^{4}$ \\ Jian Sun, ${ }^{4}$ M. Louise Herlocher, ${ }^{4}$ Alfred E. Chang, ${ }^{5}$ and Hua Yu ${ }^{1,2}$ \\ ${ }^{1}$ Immunology Program, H. Lee Moffitt Cancer Center and Research Institute, and ${ }^{2}$ Department of Medical \\ Microbiology and Immunology, University of South Florida, College of Medicine, Tampa, Florida 33612; \\ ${ }^{3}$ University of Wisconsin Comprehensive Cancer Center, Madison, Wisconsin 53792; and ${ }^{4}$ Department of \\ Epidemiology, School of Public Health, and ${ }^{5}$ Department of Surgery, School of Medicine, University of \\ Michigan, Ann Arbor, Michigan 48109.
}

\begin{abstract}
Human papillomaviruses are associated with $>90 \%$ of all cases of uterine cervical tumors. The E6 and E7 oncoproteins of human papillomavirus are potentially ideal targets of immune therapy for cervical cancer, because their expression is necessary for cellular transformation. Although both E6 and E7 proteins contain numerous predicted cytotoxic T lymphocyte (CTL) epitopes that are capable of binding to human leukocyte antigens, the majority of earlier in vivo tumor rejection studies have focused on E7. We show here that gene gun-mediated skin transfection of plasmid vector encoding the nontransforming, amino-terminal half of E6 resulted in the induction of E6-specific CTL activity and tumor rejection in a murine model. The use of recombinant murine interleukin-12 (rmIL-12) as a vaccine adjuvant has been shown to result in both an enhancement and suppression of immune responses, depending upon the doses of rmIL-12 and the experimental systems used. We demonstrate here that local expression of transgenic mIL-12 at the E6 DNA vaccination site potentiated E6-specific CTL responses and increased vaccine-induced antitumor therapeutic efficacy. Our results indicate that transfection of the mIL-12 gene at the vaccination site may represent an attractive adjuvant for cancer gene immunotherapy.
\end{abstract}

Key words: DNA vaccine; human papillomavirus type 16 E6; interleukin-12.

$\mathrm{C}$ ervical cancer accounts for $15 \%$ of all cancer-related deaths and is the second leading cause of cancer deaths in women worldwide. ${ }^{1}$ Each year $\sim 2,500,000$ women in the United States alone are diagnosed with low-grade cervical cancer precursor. ${ }^{2}$ It has been estimated that nearly 15,000 of these individuals develop cervical cancer. ${ }^{3}$ Therefore, there is a great need to develop a safe and effective vaccine for cervical cancer. The detection of human papillomavirus (HPV) DNA in $>90 \%$ of cervical tumors suggests that HPV is etiologically associated with the disease. ${ }^{4}$ HPV type 16 is the predominant HPV DNA, being observed in $40-60 \%$ of the cancers. ${ }^{4}$ Two HPV16 oncoproteins, E6 and E7, are preferentially expressed in cervical cancer cells. ${ }^{5}$ These proteins are capable of inducing transformation in cells by disrupting the functions of the cellular tumor sup-

Received May 12, 1998; accepted September 26, 1998.

Address correspondence and reprint requests to Dr. Hua Yu, Immunology Program, H. Lee Moffitt Cancer Center and Research Institute, 12902 Magnolia Drive, Tampa, FL 33612. E-mail address: huayu@moffitt.usf.edu

(C) 1999 Stockton Press 0929-1903/99/\$12.00/+0 pressors p53 and retinoblastoma. ${ }^{6-8}$ Because E6 and E7 are retained in progressive HPV-associated cervical tumors, ${ }^{5}$ they are potential tumor-specific targets for the immunotherapy of cervical cancer.

Numerous studies indicate that E6 and E7 antigens (Ags) are immunogenic in humans. Potential cytotoxic T lymphocyte (CTL) epitopes that bind to various human histocompatibility leukocyte Ags (HLAs) have been identified within the E6 and E7 proteins. ${ }^{9,10}$ Several of these epitopes were found to stimulate CTL responses in vitro in the peripheral blood mononuclear cells of patient donors. ${ }^{11,12}$ Recent clinical trials using E6 and E7 recombinant vaccinia virus ${ }^{13}$ or E7-glutathione $S$-transferase fusion proteins ${ }^{14}$ as vaccines resulted in the induction of specific CTLs in a portion of the evaluated patients. Both E6 and E7 have also been demonstrated to induce specific CTLs in animal studies. ${ }^{15-17}$ Whereas E7 has been demonstrated as a tumor-rejecting $\mathrm{Ag},{ }^{17-19}$ the ability of E6 to similarly serve as a tumor-rejection $\mathrm{Ag}$ has been much less well characterized.

Intramuscular (i.m.) or intradermal (i.d.) injection of plasmid vectors encoding viral Ags has been shown to result in the expression of the candidate therapeutic 
transgene and in the induction of Ag-specific immune responses, including antibody $(\mathrm{Ab})$, T helper, and $\mathrm{CTL}$ responses. ${ }^{20-24}$ Whereas both peptide and recombinant viral vaccines are capable of eliciting potent immune responses, a DNA-based vaccine strategy offers a number of potential advantages, such as the ability to include all encoded CTL epitopes and a lack of any infectious agents.

Effective immunogen usually requires a potent adjuvant, and recombinant murine interleukin-12 (rmIL-12) protein can be a significant antitumor adjuvant. However, systemic injection of rmIL-12 can cause severe side effects, even when used at relatively low doses. ${ }^{25}$ Furthermore, the adjuvant effect of rmIL-12 is dose-sensitive, and the dose requirement varies from one experimental system to another. ${ }^{25,26}$ Injection of high doses of rmIL-12 has also been shown to result in impaired CTL development and in higher viral titers in lymphocytic choriomeningitis-infected mice. ${ }^{27}$ High doses of rmIL-12 treatment can also lead to transient immune suppression prior to any rmIL-12-enhanced antitumor adjuvant effect. ${ }^{26}$ The antitumor adjuvant effect is mediated by the ability of rmIL-12 to inhibit angiogenesis rather than by the stimulation of Ag-specific immune responses. ${ }^{28} \mathrm{We}$ reported previously that an i.d. injection of cDNAencoding IL-12 resulted in local expression of the mIL-12 transgene and in an induction of systemic immune responses, including interferon- $\gamma($ IFN- $\gamma$ ) production by cultured splenocytes and an augmentation of natural killer (NK) lytic activity as well as the inhibition of tumor growth, without detectable side effects. ${ }^{29} \mathrm{We}$ report here that gene gun-mediated in vivo skin transfection of a nontransforming E6 mutant gene can lead to the induction of E6-specific CTLs, resulting in tumor rejection in $\mathrm{BALB} / \mathrm{c}$ mice. Furthermore, the expression of an IL-12 transgene at the immunization site enhances Ag-specific immune responses, including CTL activation, as well as tumor rejection.

\section{MATERIALS AND METHODS}

\section{Plasmid expression vectors}

Primers containing convenient restriction enzyme sites were synthesized to generate polymerase chain reaction (PCR) products encoding the entire E6-E7 sequences or an E6 deletion mutant encoding only the first 63 amino acids. For both E6-E7 and E6 PCRs, the forward primer was 5'CGAGAATTCAGCAGACATTTTATGCACCA (corresponding to HPV16 nucleotides 71-91), which included the translation initiation codon for E6 (underlined). An EcoRI site (bold) was also included in the primer for cloning purposes. The reverse primer for E6-E7 cloning was 5'-CTGGATCCTTATGGTTTCTGAGAACAGATGGGGCCCACAATTCC, which corresponds to the HPV nucleotides $832-868$ in addition to a Bam HI site (bold). PCR amplification of the E6-E7 sequences was performed as described previously. ${ }^{29}$ A plasmid containing the entire HPV16 genome (generously provided by Dr. T. Carey of the University of Michigan) was used as a template for the PCR. The PCR products were digested with EcoRI and BamHI and gel-purified before being inserted into pSG5 (Stratagene, La Jolla, Calif). To clone the E6-E7 fragment into
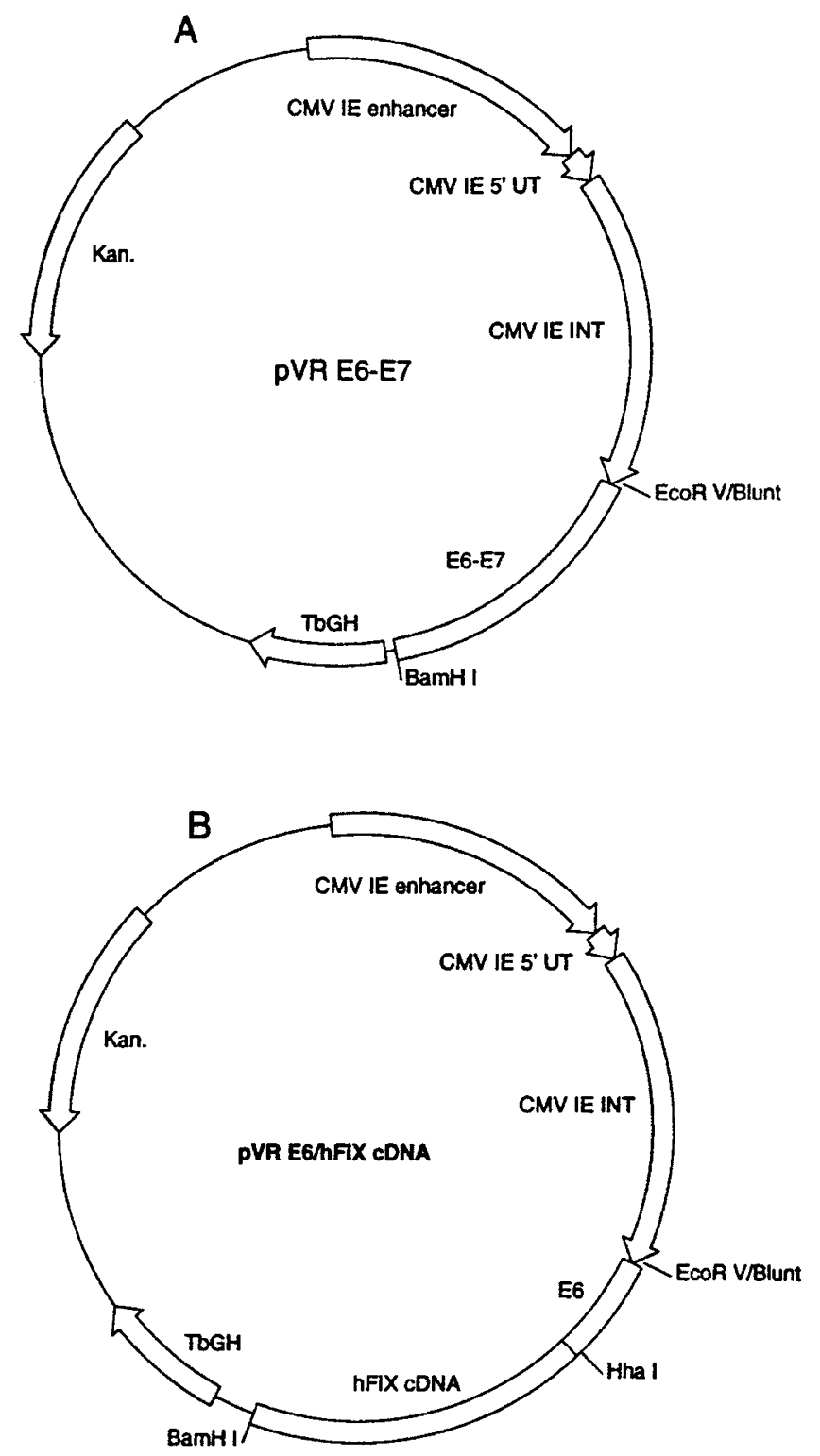

Figure 1. E6-expressing plasmid constructs. Details of the construction are described in Materials and Methods. pVRE6-E7 contains the HPV16 sequences encoding full-length E6 and E7. This construct was used to transfect Renca tumor cells. pVRE6hFIX contains the first 186 nucleotides of the E6 gene encoding the amino-terminal half of the E6 protein. The hFIX CDNA in the construct lacks the translation initiation codon (ATG). pVRE6hFIX plasmid was used for DNA vaccination experiments.

a high expression vector, pVR1012 (kindly provided by Vical, San Diego, Calif), pSG5E6-E7 was digested with EcoRI, blunt-ended, and subsequently cut with BamHI and inserted into pVR1012 at EcoRV and BamHI. The resulting pVR1012E6-E7 (pVRE6-E7) is shown in Figure 1A. To amplify the HPV16 sequences encoding the amino-terminal half of the E6 open reading frame, another PCR was performed. The forward primer was the same as that used for E6-E7 amplification. The reverse primer (5'-CGTGCGCCTATATACTATGCATAAATCCCG) contained an Hha I site (bold) and HPV16 E6 sequences corresponding to nucle- 
otides 244-267. The amplified E6 (coding sequences 1-186) PCR fragment was digested with EcoRI and HhaI. The human factor IX (hFIX) cDNA (a generous gift of Dr. K. Kurachi, University of Michigan) was digested with Hha I and BamHI. The restriction enzyme-digested E6 and hFIX fragments were ligated into EcoRI- and BamHI-digested pSG5. The translation initiation codon ATG was deleted in the Hha I and BamHI fragment of hFIX. The resulting final construct encoded an E6 (amino terminus)-hFIX fusion protein. Although the E6 product is not normally secreted, the hFIX cDNA product contains a signal endoplasmic reticulum insertional sequence. When this construct was transfected into Renca cells, the hFIX protein was readily detectable in the culture supernatants as indicated by an enzyme-linked immunosorbent assay (ELISA) (data not shown). This fusion protein construct was later used as an indicator for the translation of the E6 mutant protein in vivo by assaying the serum levels of hFIX protein. pSGE6hFIX was converted to pVRE6hFIX by the same method as the pSG5E6-E7 to pVRE6-E7 conversion described above.

The luciferase and mIL-12 cDNA expression vectors used in this study were described previously. ${ }^{30}$

\section{Tumor cells and mice}

Renca tumor cells (kindly provided by Dr. R. Wiltrout, National Cancer Institute, Frederick, Md) were grown in RPMI 1640 medium supplemented with $10 \%$ fetal bovine sera, $2 \mathrm{mM}$ glutamine, $1 \mathrm{mM}$ sodium pyruvate, $1 \%$ minimal Eagle's medium nonessential amino acids, $100 \mathrm{IU} / \mathrm{mL}$ penicillin, and 50 $\mu \mathrm{g} / \mathrm{mL}$ gentamicin. BALB/c female mice were purchased from the National Cancer Institute. The mice were housed in the pathogen-free animal facility at the H. Lee Moffitt Cancer Center and Research Institute at the University of South Florida. Cohorts of 6- to 8-week-old BALB/c female mice were used for the experiments.

\section{Tumor cell transfection and expression of pVRE6-E7}

Transfections of cultured cells were carried out by the lipofectamine-mediated method. G418 resistance was provided by cotransfecting $\mathrm{pSV}_{2}$-neo with $\mathrm{pVRE6-E7}$ at a 1:10 molar ratio. The expression of E6 was determined by reverse transcriptase (RT)-PCR as described previously, ${ }^{29}$ and the primers used were the same as the ones used to generate the E6 deletion mutant gene described above. Human CaSki cells, which are positive for HPV16, and human keratinocytes (HKC) transfected with HPV16 genomic DNA ${ }^{31}$ were used as positive controls for E6 RNA detection. HPV16-transfected HKC cells and CaSki cells were kindly provided by Dr. T. Munoz-Antonia of the H. Lee Moffitt Cancer Center and Research Institute.

\section{DNA vaccination and tumor challenge}

Plasmid DNAs were prepared by banding twice in $\mathrm{CsCl}_{2}$ gradients. Gene gun-mediated DNA transfection was essentially as described previously. ${ }^{30}$ The Accell helium-driven gene gun was kindly provided by PowderJect (Middleton, Wis). Briefly, plasmid DNAs were precipitated onto gold beads and coated onto the inner surface of Tefzel tubing (McMaster, Atlanta, Ga). Each transfection consisted of $0.5 \mathrm{mg}$ of $2-\mu \mathrm{m}$ gold beads and $1.25 \mu \mathrm{g}$ of DNA as described previously. ${ }^{30}$ Cotransfection with pVRhFIXE6 and pIL-12 (IL-12 expression vector) contained the same amount of total plasmid DNAs at a 1:1 ratio by weight. The abdomens of mice were shaved, and plasmid DNAs were delivered by the gene gun to the skin at four abdominal positions with minimal overlap. To determine the levels of IL-12 transgene expression in vivo, either the IL-12 expression vector or a luciferase expression vector (negative control) was delivered. For both tumor challenge experiments and CTL assays, two vaccinations, either 1 or 2 weeks apart, were performed. Subcutaneous tumor challenges involving $2 \times 10^{5}$ E6-E7 Renca cells or wild-type (wt) Renca cells were injected at the abdomen either 1 or 2 weeks after the second vaccination. Tumor growth was monitored three times per week by measuring two perpendicular tumor diameters with a caliper. To minimize discomfort, mice were euthanized when tumors abscessed through the skin or reached a diameter of $10 \mathrm{~mm}$.

\section{ELISAS}

To determine the serum levels of hFIX, a sandwich ELISA was performed. Medium-bind enzyme immunoassay plates (Costar, Cambridge, Mass) were coated with monoclonal anti-hFIX Ab (1 $\mu \mathrm{g} / \mathrm{mL})$ (Hematologic Laboratories, Essex Junction, $\mathrm{Vt}$ ), incubated overnight at $4^{\circ} \mathrm{C}$, and blocked for 30 minutes. A serial dilution of pooled human blood (George King Biomedical, Overland Park, Kan) and samples were incubated for 2 hours, followed by rabbit anti-hFIX Ab (1 $\mu \mathrm{g} / \mathrm{mL}$ ) (Sigma Immuno Chemicals, St. Louis, Mo). Horseradish peroxidase-conjugated goat anti-rabbit immunoglobulin $G$ (1/1000 dilution) was added, followed by 2,2-azino-di(3-ethylbenzothiazoline sulfonate) substrate solution. Plates were developed for 30 minutes, and absorbance was measured at $450 \mathrm{~nm}$.

To determine the in vivo expression levels of IL-12 both at the skin transfection site and in sera, a sandwich ELISA was performed according to the manufacturer's suggestions (Genzyme, Cambridge, Mass). Serum samples and protein extracts from transfected skin tissues were obtained and prepared as described previously ${ }^{30}$ at $8,24,72$, and 120 hours posttransfection.

\section{Cytotoxicity assays}

Single-cell suspensions of splenocytes were prepared as described previously ${ }^{29}$ from mice that has been injected with either the hFIX plasmid DNA (control vector), pVRE6hFIX, or E6/IL-12 expression vectors. Cytotoxic lytic activity was assayed 5 days after an in vitro restimulation with $5 \times 10^{4}$ irradiated E6-E7 Renca cells (3500 rad). ${ }^{51} \mathrm{Cr}$ labeling and a 4-hour ${ }^{51} \mathrm{Cr}$ release assay were performed as described previously. ${ }^{29,30}$ Both ${ }^{51} \mathrm{Cr}$-labeled wt Renca cells (negative control) and E6-E7 Renca cells were used as target cells, respectively. Total release represents the radioactivity released after lysis of target cells with 5\% Triton X-100.

\section{Statistical analysis}

Cox proportional hazard regressions were performed on the mice challenged with E6-E7 Renca and Renca tumors. In both cases, time until tumor growth was modeled as a function of E6 and IL-12 (0/1 variables). For mice challenged with E6-E7 Renca, the hazard ratios associated with the E6 and IL-12 vaccinations were 0.017 and 0.020 , respectively. Both were highly significant $(P<.0001)$. For mice challenged with parental Renca tumors, the hazard ratio associated with E6 immunization was 0.606 , which was not statistically significant $(P=.1669)$. The hazard ratio for the IL-12 immunization was 0.372 , which was significant $(P=.0072)$. 

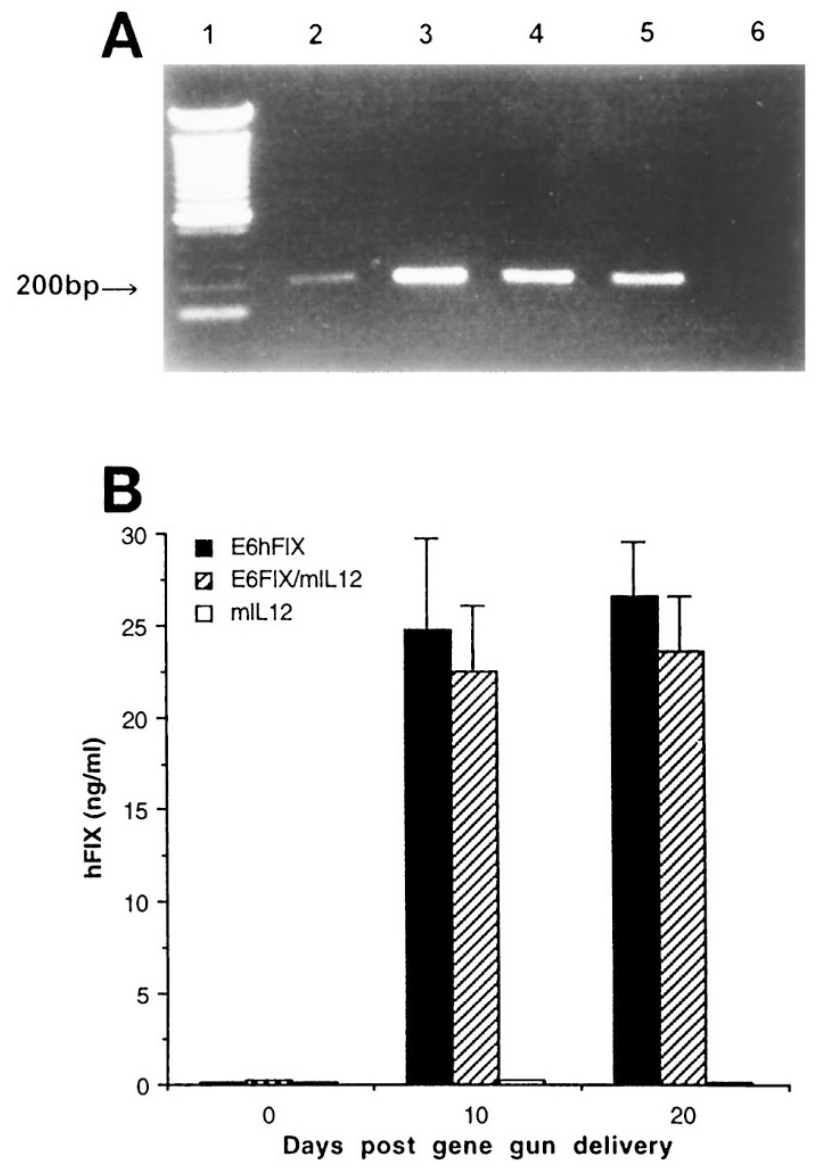

Figure 2. Expression of E6 in E6-E7 Renca cells and expression of E6hFIX transgene in vivo after gene gun-mediated skin transfection. A: RT-PCR analysis of total RNA from E6-E7 Renca clones. Lane 1: 100-bp ladder; lanes 2 and 3: cDNA prepared from two E6-E7 Renca clones; lanes 4 and 5, cDNA prepared from HPV16-transfected HKC cells and CaSki cells, respectively, as positive controls; lane 6, cDNA isolated from Renca cells (E6-negative). B: ELISA of $\mathrm{hFIX}$ as an indicator of the translation of the E6 fusion protein. Two mice per experimental group were tail-bled at 0,10 , and 20 days after DNA transfection. An ELISA using the mouse sera was performed as described in Materials and Methods.

\section{RESULTS}

Expression of $p$ VRE6-E7, $p$ VRE6hFIX, and $m I L-12$ cDNA vectors

To generate E6-expressing tumor cells for tumor challenge, Renca cells were transfected with pVRE6-E7 (Fig 1A) and $\mathrm{pSV}_{2}$-neo. To obtain E6-expressing Renca clones, G418-resistant Renca clones derived from pVRE6-E7/ $\mathrm{pSV}_{2}$-neo cotransfection were screened by RT-PCR for E6 expression. An 200-base pair (bp) band was detected in both E6-E7 Renca clones and the two HPV16-positive cell lines, but not in wt Renca cells (Fig 2A).

To generate an E6 mutant DNA vaccine that lacked oncogenic transforming capacity but retained most of the predicted immunogenic portions, we chose to insert E6 coding sequences (1-186 bp) into the expression vector. Mutational analysis implicated domains in the carboxyl-terminal half of the E6 protein in transformation. ${ }^{32,33}$ Studies on peptide binding to HLAs identified several potential CTL epitopes in the E6 amino-terminal half, including all of the epitopes with high affinity to HLA-A $2,{ }^{9}$ the most common HLA alleles present in $\sim 50 \%$ of the overall population.

To facilitate detection of the in vivo expression of E6 at the protein level after DNA vaccination, hFIX cDNA was included in the E6 deletion mutant expression vector (Fig 1B) to generate an in-frame fusion protein. Because the hFIX cDNA lacked a translation initiation codon and was placed at the $3^{\prime}$ end of the E6 aminoterminal coding region, the detection of the hFIX protein should then be indicative of E6 transgene translation. An ELISA specific for detecting hFIX protein in mouse sera was performed at days 0,10 , and 20 after gene gun-mediated skin transfections of the vectors. As shown in Figure 2B, mice that had been DNA-vaccinated with either $\mathrm{pVRE6hFIX} \mathrm{or} \mathrm{pVRE6hFIX/pIL-12}$ vectors were serum-positive for hFIX transgenic production at days 10 and 20, whereas mice that received the IL-12 vector alone were negative. Therefore, in the mice injected with pVRE6hFIX plasmid, the E6 mutant protein is most likely translated, because the hFIX portion of the transgenic fusion protein was being expressed correctly.

To determine the levels of expression of IL-12 transgene at the protein level, mIL-12-specific ELISAs were performed. Both transfected skin tissues and serum samples were evaluated in the assay. The results (Fig 3 ) showed that IL-12 transgene expression at 8 and 24 hours posttransfection was readily detectable at vaccination sites, whereas systemic levels of IL-12 protein in sera were 50 - to 100 -fold lower.

DNA vaccination with the $5^{\prime}$ half of the HPV16 E6 gene induces E6-specific tumor rejection that is potentiated by cotransfection with IL-12 CDNA

To determine whether vaccination with the mutant E6 expression vector could lead to E6-specific tumor rejection, mice were transfected epidermally with either the phFIX vector or $\mathrm{pVRE6hFIX} \mathrm{vector,} \mathrm{followed} \mathrm{by} \mathrm{tumor}$ challenge. Both groups of mice were challenged with either $2 \times 10^{5}$ E6-E7 Renca or wt Renca cells. As shown in Figure 4A, E6 DNA vaccination inhibited E6-expressing tumor growth in all of the mice tested and elicited tumor rejection in $30 \%$ of the mice. Data in Figure 4A represent 20 mice per group (with the exception of the hFIX control group, which included 15 mice) pooled from three independent experiments. The delay of tumor emergence in pVRE6hFIX-immunized mice compared with those immunized with phFIX is highly significant $(P<.0001)$ (Fig 4A). In contrast, $90 \%$ of the mice immunized with the E6 vector failed to reject the E6-negative, parental tumors (Fig 4B). Although one of the pE6hFIX-immunized mice did not develop any tumor, the kinetics of tumor onset in mice immunized with IL-12 in the absence or presence pE6hFIX were 

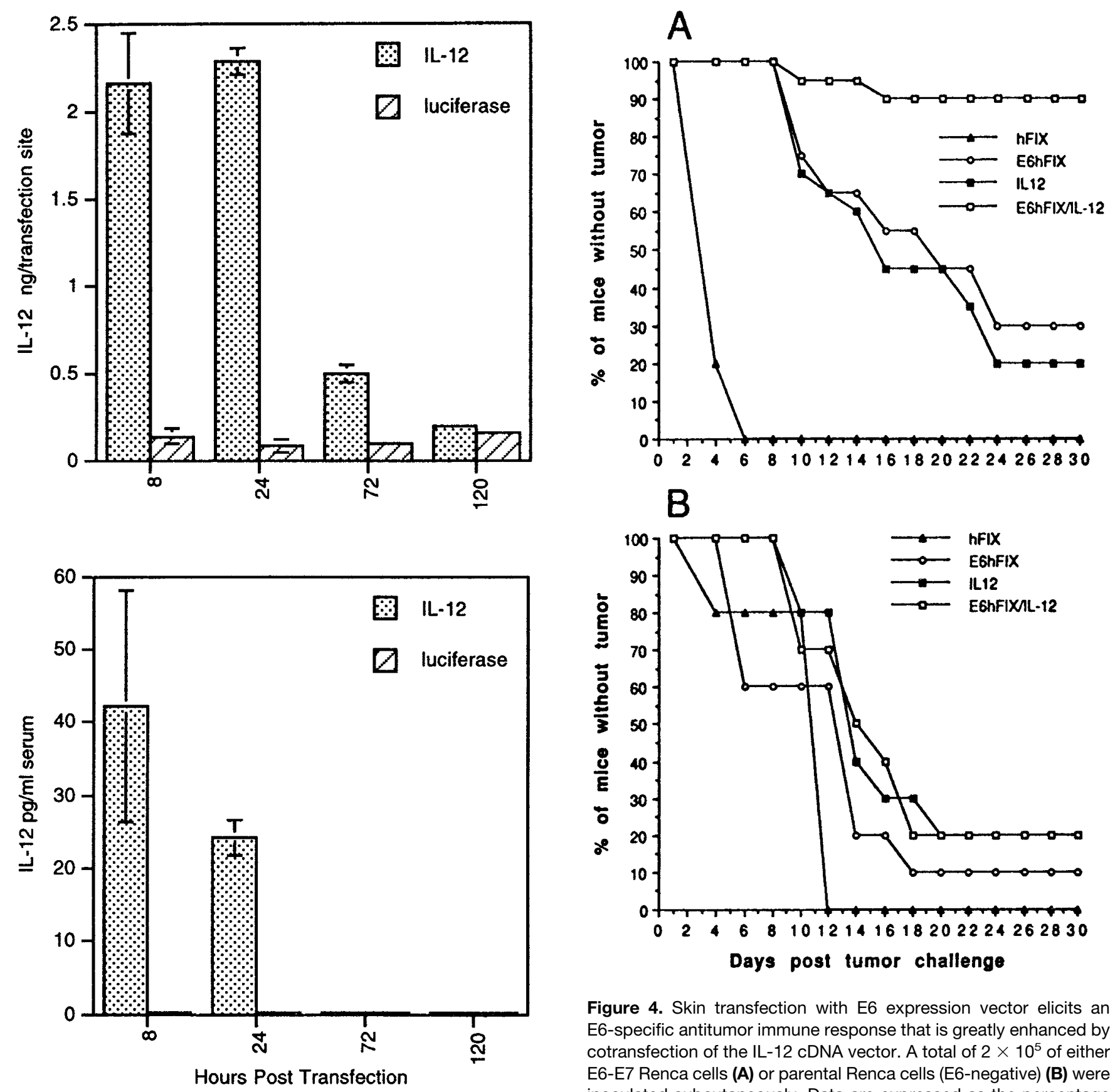

Figure 3. ELISA of IL-12 cytokine in transfected skin tissues and in sera of mice that had received DNA transfections. Two individual mice per group were assayed at each timepoint. The same two mice at every timepoint were assayed for both skin tissue and serum levels. Luciferase cDNA vector ${ }^{30}$ transfection was performed sideby-side with IL-12 cDNA transfection and was used here as a negative control.

similar (Fig 4B). The delay of E6-negative tumor onset observed in mice immunized with pE6hFIX in the absence and presence of IL-12 compared with those immunized with phFIX and IL-12, respectively, is not statistically significant $(P=.1669)$ (Fig 4B). Therefore the data shown here suggest that the antitumor effect

Figure 4. Skin transfection with E6 expression vector elicits an E6-specific antitumor immune response that is greatly enhanced by cotransfection of the IL-12 cDNA vector. A total of $2 \times 10^{5}$ of either E6-E7 Renca cells (A) or parental Renca cells (E6-negative) (B) were inoculated subcutaneously. Data are expressed as the percentage of tumor-free animals. The mice were terminated at day 30 .

shown in Figure 4A as a result of E6 DNA vaccination is E6-specific.

Although E6-specific antitumor immunity was observed after gene gun-mediated skin transfer of the E6 expression vector, the efficacy of the antitumor effect was low (30\% tumor-free) (Fig 4A). We have shown previously that an i.d. injection of IL-12 cDNA resulted in the induction of splenocyte IFN- $\gamma$ production and NK activity as well as in an inhibition of tumor growth at a distant site. ${ }^{29}$ To evaluate whether cotransfection of IL-12 cDNA with E6 DNA could enhance E6-specific 

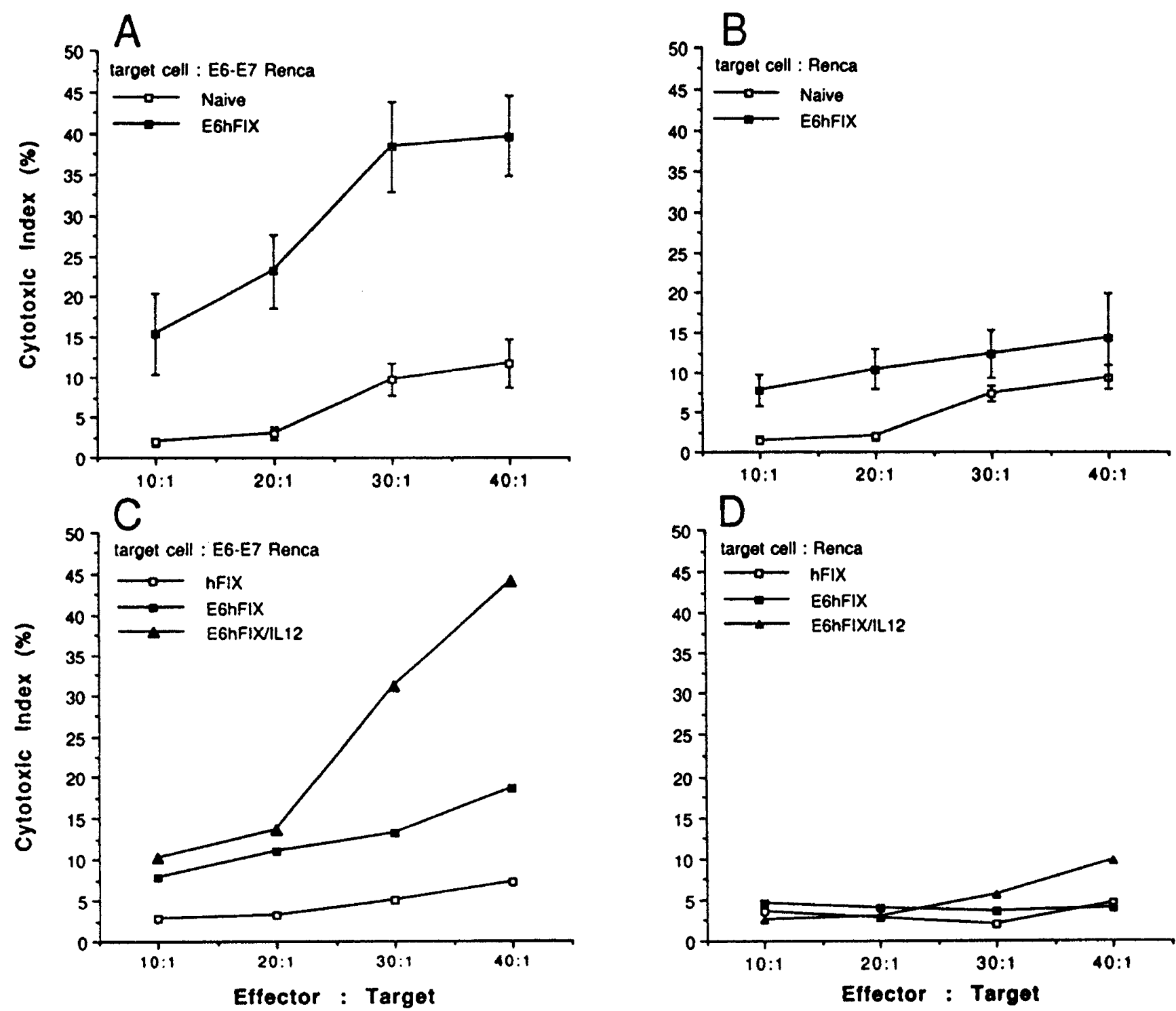

Figure 5. Vaccination with pVRE6hFIX DNA results in E6-specific CTLs, and the E6 CTL is potentiated by cotransfection with the IL-12 cDNA vector. Splenocytes were prepared from mice after the second immunization. The data in $\mathbf{A}$ and $\mathbf{B}$ were obtained from splenocytes prepared from the same mice and represent the means of two mice per group; experiments were repeated once with similar results. The results shown in $\mathbf{C}$ and $\mathbf{D}$ also used splenocytes prepared from the same mice, represent pooled samples of three mice per group, and were confirmed by two additional experiments. ${ }^{51} \mathrm{Cr}$ release assays were performed after in vitro restimulation with irradiated E6-E7 Renca cells for 5 days. The target cells used for ${ }^{51} \mathrm{Cr}$ release assays were E6-E7 Renca $(\mathbf{A}, \mathbf{C})$ and Renca (B,D), respectively.

tumor rejection, IL-12 and E6 expression vector DNAs were coprecipitated onto gold beads for gene gunmediated skin DNA delivery. Compared with vaccination with either pVRE6hFIX or IL-12 vectors alone, pVRE6hFIX/IL-12 in combination elicited markedly stronger antitumor immunity $(30 \%, 20 \%$, and $90 \%$ tumor rejection, respectively; Fig 4A). To determine whether the increased antitumor effect was a result of anti-angiogenesis mediated by IL-12 transgene expression, a Matrigel/hemoglobin assay to evaluate angiogenesis was performed. No reduction of angiogenesis was observed in mice treated with IL-12 cDNA in vivo skin transfection (data not shown).
E6-specific CTL is potentiated by cotransfection with $I L-12$ expression vector at the vaccination site

Previous studies demonstrated that immunization with E6 recombinant vaccinia virus could elicit E6-specific CTL responses in both $\mathrm{C} 57 \mathrm{BL} / 6\left(\mathrm{H}-2^{\mathrm{b}}\right)$ and $\mathrm{DBA} / 2$ $\left(\mathrm{H}-2^{\mathrm{d}}\right)$ mice. $^{15}$ To determine whether vaccination with mutant E6 DNA could also induce E6-specific CTL responses in $\mathrm{BALB} / \mathrm{c}\left(\mathrm{H}-2^{\mathrm{d}}\right)$ mice, spleen cells from mice transfected with either phFIX or pVRE6hFIX were assayed for CTL activity. An E6-specific CTL response was elicited by DNA immunization with the E6 expression vector (Fig 5, A and B). When the IL-12 
expression vector DNAs were transfected with E6 expression vectors for immunizations, the CTL response against E6-E7 Renca was significantly enhanced (Fig 5C). When E6-negative Renca cells were used as target cells, no significant CTL lysis was observed (Fig 5D).

\section{DISCUSSION}

Although HPV16 E6 has been shown to be selectively retained in a majority of the progressed cervical tumors, ${ }^{5}$ and numerous predicted CTL epitopes capable of binding various HLAs have been identified in the $\mathrm{Ag}$ in vitro, ${ }^{9}$ many of the earlier in vivo tumor rejection studies have focused on E7, which is coretained with E6 in the transformed cells. ${ }^{17-19}$ Our current study provides evidence that the nontransforming amino-terminal half of HPV16 E6 contains tumor rejection epitopes in BALB/c mice. Previous studies also showed that the immunization of mice with E6-expressing recombinant vaccinia virus elicited specific $\mathrm{Ab}$, proliferative, and CTL responses. ${ }^{15}$ Consistent with these findings, our DNAbased vaccine also induced E6-specific CTL responses.

Currently, several clinical trials are underway using vaccine consisting of either E7 peptide (HLA-A2-binding) vaccine, E7-glutathione $S$-transferase fusion protein, or recombinant E6/E7 vaccinia virus. ${ }^{14}$ Although it is too early to draw conclusions from the clinical trials, CTL responses have been detected in a portion of the evaluated patients. ${ }^{14}$ Several studies have shown that some cervical cancer patients still retain natural CTL precursors against HPV16 E6 and E7, ${ }^{11,12}$ suggesting that immunotherapy based on HPV16 E6/E7 vaccine(s) might be effective in these patients.

DNA-based vaccines (direct needle injection or gene gun-mediated transfection) have been shown to successfully elicit both humoral and cellular immunity against various infectious agents. ${ }^{20-24}$ These two DNA-based approaches have also been demonstrated to induce various antitumor immune responses. ${ }^{34,35}$ Although gene gun-mediated skin transfer requires DNA precipitation onto gold beads, whereas direct needle injection only involves saline, gene gun-mediated DNA vaccination apparently results in qualitatively and quantitatively different immune responses than an i.m. injection ${ }^{36}$ and apparently, under certain circumstances, can be more efficacious than a direct i.m. injection in inducing similar immune responses. ${ }^{21,22}$ Compared with peptide-based vaccines, DNA vaccine may be advantageous in that plasmid purification is much easier than peptide synthesis and, unlike peptides, DNA encoding numerous CTL epitopes can be included in a single expression vector. The amino-terminal half of E6 encoded by the E6 gene in our vaccine vector, for example, is expected to contain all of the potential CTL epitopes exhibiting high binding affinity to HLA-A2.1. Immunizations with live attenuated or recombinant viruses have been successfully used for the prevention of a number of infectious diseases and have been shown to be an effective approach for cancer therapy in animal models. However, ease of purification and production of plasmid DNAs make DNA-based vaccine strategy an attractive alternative to viral vector systems/vaccines for the prevention of infectious diseases or for cancer immunotherapy.

In this study, vaccinating mice with the E6 expression vector alone elicited detectable CTL responses and significant tumor inhibition/rejection. Whereas some delay of tumor emergence was observed in mice vaccinated with pVRE6hFIX compared with those vaccinated with phFIX when challenged with parental Renca tumor cells, no difference in tumor emergence was observed between mice immunized with E6hFIX/IL-12 and IL-12 when challenged with Renca (Fig 4B). In addition, little lysis of Renca cells by splenocytes derived from E6-immunized mice was observed (Fig 5, B and D). Taken together, whereas vaccination with pVRE6hFIX may result in slight, insignificant nonspecific immune responses, it is clear the E6 DNA vaccine can elicit E6-specific antitumor immunity.

Although using rmIL-12 as an adjuvant to potentiate antitumor responses has been documented, rmIL-12 can also exert inhibitory effects on the tumor Ag-specific responses induced by tumor cell vaccination. A study by Kurzawa et al demonstrated that rmIL-12 induced transient immune suppression at 14 days after tumor cell vaccination/rmIL-12 treatment. ${ }^{26}$ Immune suppression included reduced splenic CTLs, impaired cytokine (i.e., IFN- $\gamma$ ) secretion from splenocytes, and the ability to reject tumor challenge. By day 28 , rmIL-12 treatment was shown to enhance the tumor rejection induced by the tumor cell/GM-CSF vaccine. The authors suggest that Ag-nonspecific antitumor mechanisms (e.g., antiangiogenesis) are involved in mediating the IL-12 adjuvant effect. ${ }^{26,28}$ In our system, we were unable to detect a reduction of angiogenesis at Renca tumors injected 7 days after IL-12 cDNA skin transfection (data not shown). Another study in which rmIL-12 was used as an adjuvant for cancer vaccine involved the p53 peptides. ${ }^{25}$ When used at very low doses ( $1 \mathrm{ng} /$ day), rmIL-12 significantly potentiated the p53-specific CTL response and tumor rejection. At higher doses of rmIL-12 (10$100 \mathrm{ng}$ /day), however, the adjuvant effect was diminished. In the study by Noguchi et al, $1 \mathrm{ng} /$ day rmIL-12 treatment did not lead to immune suppression, nor did it have any adjuvant effect. ${ }^{25}$ These studies suggest that low doses of rmIL-12 can enhance tumor Ag-specific immune responses. However, different Ags and vaccination procedures may require different doses of rmIL-12.

In our study, low levels of mIL-12 transgene expression were readily detectable at the immunization site (2 $\mathrm{ng} / \mathrm{site}$ ), and the peak serum level of IL-12 after IL-12 cDNA transfection was $<50 \mathrm{pg} / \mathrm{mL}$ (Fig 3B). We have shown previously that the transgenic expression of IL-12 after in vivo IL-12 skin transfection alone resulted in the induction of splenic IFN- $\gamma$ secretion and NK lytic activity. ${ }^{29}$ In the current study, transgenic expression at the vaccination site of IL-12 enhances CTL activity and tumor rejection at both 1 and 3 weeks after a second immunization. A recent study by Kim et al demonstrated that i.m. coinjection of IL-12 cDNA with an HIV DNA 
immunogen resulted in elevated, Ag-specific CTLs (at 21 days after the first immunization), although no IL-12 adjuvant effect on tumor rejection was determined due to the nature of the experimental system. ${ }^{37}$ Together with these studies, our current work suggests that low levels of local transgenic expression of IL-12 at the vaccination site result in enhanced $\mathrm{Ag}$-specific immune responses, including CTLs and protective antitumor immunity.

\section{ACKNOWLEDGMENTS}

We thank PowderJect (Middleton, Wis) for providing the gene gun and Vical for the pVR1012 expression vector. We gratefully acknowledge Dr. Alan Cantor of the Biostatistics Core at the H. Lee Moffitt Cancer Center for statistical analysis. We also thank Drs. Walter Storkus, William Lee, and Catherine Newton for critical reading of the manuscript. This work was funded in part by grants from the National Cancer Institute (CA75243-01) and the University of South Florida (to H.Y.).

\section{REFERENCES}

1. Parkin DM, Stjernsward J, Muir CS. Estimates of the worldwide frequency of twelve major cancers. Bull World Health Organ. 1984;62:162-182.

2. Kurman RJ, Henson DE, Herbst AL, et al. Interim guidelines for management of abnormal cervical cytology: the 1992 National Cancer Institute Workshop. JAMA. 1994;271:1866-1869.

3. Parker SL, Tong T, Bolden S, et al. Cancer statistics. $C A$ Cancer J Clin. 1997;47:5-27.

4. Lowry DR, Kirnbauer R, Schiller JR. Genital human papillomavirus infection. Proc Natl Acad Sci USA. 1994;91: 2436-2440.

5. von Knebel Doeberitz M, Rittmuller C, Aengeneyndt F, et al. Reversible repression of papillomavirus oncogene expression in cervical carcinoma cells: consequences for phenotype and E6-p53 and E7-pRB interactions. J Virol. 1994;68:2811-2821.

6. Scheffner M, Werness BA, Huibregtse JM, et al. The E6 oncoprotein encoded by human papillomavirus type 16 and 18 promotes the degradation of p53. Cell. 1990;63: 1129-1136.

7. Werness BA, Levine AJ, Howley PM. Association of HPV-16 and HPV-18 E6 oncoproteins with the cellular P53 protein in vitro. Science. 1990;248:76-79.

8. Dyson N, Howley PM, Munger K, et al. The human papilloma virus-16 E7 oncoprotein is able to bind to the retinoblastoma gene product. Science. 1989;243:934-937.

9. Kast WM, Brandt RMP, Sidney J, et al. Role of HLA-A motifs in identification of potential CTL epitopes in human papillomavirus type $16 \mathrm{E} 6$ and E7 proteins. J Immunol. 1994;152:3904-3912.

10. Bartholomew JS, Stacey SN, Coles B, et al. Identification of a naturally processed HLA A0201-restricted viral peptide from cells expressing human papillomavirus type 16 E6 oncoprotein. Eur J Immunol. 1994;24:3175-3179.

11. Ressing ME, van Driel WJ, Celis E, et al. Occasional memory cytotoxic T-cell responses of patients with human papillomavirus type 16-positive cervical lesions against a human leukocyte antigen-A*0201-restricted E7-encoded epitope. Cancer Res. 1996;56:582-588.
12. Evans C, Bauer S, Grubert T, et al. HLA-A2-restricted peripheral blood cytolytic T lymphocyte response to HPV type 16 proteins E6 and E7 from patients with neoplastic cervical lesions. Cancer Immunol Immunother. 1996;42: 151-160.

13. Borysiewicz LK, Fiander A, Nimako M, et al. A recombinant vaccinia virus encoding human papillomavirus type 16 and 18 E6 and E7 proteins as immunotherapy for cervical cancer. Lancet. 1966;347:1523-1527.

14. Tindle RW. Human papillomavirus vaccines for cervical cancer. Curr Opin Immunol. 1996;8:643-650.

15. Gao L, Chain B, Sinclair C, et al. Immune response to human papillomavirus type 16 E6 gene in a live vaccinia vector. J Gen Virol. 1994;75:157-164.

16. Gao L, Walter J, Travers P, et al. Tumor-associated E6 protein of human papillomavirus type 16 contains an unusual $\mathrm{H}-2 \mathrm{~Kb}$-restricted cytotoxic T cell epitope. J Immunol. 1995;155:5519-5526.

17. Feltkamp MCW, Smits HL, Vierboom MPM, et al. Vaccination with cytotoxic $\mathrm{T}$ lymphocyte epitope-containing peptide protects against a tumor induced by human papillomavirus type 16-transformed cells. Eur J Immunol. 1993;23:2242-2249.

18. Chen L, Thomas EK, Hu S-L, et al. Human papillomavirus type 16 nucleoprotein E7 is a tumor rejection antigen. Proc Natl Acad Sci USA. 1991;88:110-114.

19. Wu T-C, Guarnieri FG, Staveley-O'Carroll KF, et al. Engineering an intracellular pathway for major histocompatibility complex class II presentation of antigens. Proc Natl Acad Sci USA. 1995;92:11671-11675.

20. Ulmer JB, Donnelly JJ, Parker SE, et al. Heterologous protection against influenza by injection of DNA encoding a viral protein. Science. 1993;259:1745-1749.

21. Pertmer TM, Eisenbraun MD, McCabe D, et al. Gene gun-based nucleic acid immunization: elicitation of humoral and cytotoxic $\mathrm{T}$ lymphocyte responses following epidermal delivery of nanogram quantities of DNA. Vaccine. 1995; 13:1427-1430.

22. Fynan E, Webster RG, Fuller DH, et al. DNA vaccines: protective immunizations by parental, mucosal, and genegun inoculations. Proc Natl Acad Sci USA. 1993;90:1147811482.

23. Sedegah M, Hedstrom R, Hobart P, et al. Protection against malaria by immunization with plasmid DNA encoding circumsporozoite protein. Proc Natl Acad Sci USA. 1994;91:9866-9870.

24. Wang B, Merva M, Dang K, et al. DNA inoculation induces proliferative in vivo immune responses against cellular challenge with HIV-1 antigen-expressing cells. AIDS Res Hum Retroviruses. 1994;10:S35-S41.

25. Noguchi Y, Richards EC, Chen Y-T, et al. Influence of interleukin 12 on p53 peptide vaccination against established Meth A sarcoma. Proc Natl Acad Sci USA. 1995;92: 2219-2223.

26. Kurzawa H, Wysocka M, Aruga E, et al. Recombinant interleukin IL-12 enhances cellular immune responses to vaccination only after a period of suppression. Cancer Res. 1998;58:491-499.

27. Orange JS, Wolf SF, Biron CA. Effects of IL-12 on the response and susceptibility to experimental viral infections. J Immunol. 1994;152:1253-1264.

28. Coughlin CM, Salhany KE, Wysocka M, et al. Interleukin-12 and interleukin-18 synergistically induce murine tumor regression which involves inhibition of angiogenesis. J Clin Invest. 1998;101:1441-1452.

29. Tan J, Newton CA, Djeu J, et al. Injection of cDNA 
encoding interleukin-12 inhibits tumor establishment at a distant site in a murine renal carcinoma model. Cancer Res. 1996;56:3399-3403.

30. Rakhmilevich AL, Turner J, Ford MJ, et al. Gene gunmediated skin transfection with interleukin 12 gene results in regression of established primary and metastatic murine tumors. Proc Natl Acad Sci USA. 1996;93:6291-6296.

31. Pirisi L, Creek KE, Doniger J, et al. Continuous cell lines with altered growth and differentiation properties originate after transfection of human keratinocytes with human papillomavirus type 16 DNA. Carcinogenesis. 1988;9:15731579.

32. Pim D, Storey A, Thomas M, et al. Mutational analysis of HPV-18 E6 identifies domains required for p53 degradation in vitro, abolition of 533 transactivation in vivo, and immortalization of primary BMK cells. Oncogene. 1994;9: 1869-1872.

33. Dalal S, Gao EJ, Androphy EJ, et al. Mutational analysis of human papillomavirus type 16 E6 demonstrates that p53 degradation is necessary for immortalization of mammary epithelial cells. J Virol. 1996;70:683-688.

34. Conry RM, LoBuglio AF, Loechel F, et al. A carcinoembryonic antigen polynucleotide vaccine has in vivo antitumor activity. Gene Ther. 1995;2:59-65.

35. Schirmbeck R, Bohm W, Teimann J. DNA vaccination primes MHC class I-restricted, simian virus 40 large tumor antigen-specific CTL in $\mathrm{H}-2^{\mathrm{d}}$ mice that reject syngeneic tumors. J Immunol. 1996;157:3550-3558.

36. Feltquate DM, Heaney S, Webster RG, et al. Different Th cell types and antibody isotypes generated by saline and gene gun DNA immunization. J Immunol. 1997;158:22782284.

37. Kim JJ, Ayyavoo V, Bagarazzi ML, et al. In vivo engineering of a cellular immune response by coadministration of IL-12 expression vector with a DNA immunogen. J Immunol. 1997;158:816-826. 